Network Working Group

Request for Comments: 4818

Category: Standards Track
J. Salowey

R. Droms

Cisco Systems, Inc.

April 2007

RADIUS Delegated-IPv6-Prefix Attribute

Status of This Memo

This document specifies an Internet standards track protocol for the Internet community, and requests discussion and suggestions for improvements. Please refer to the current edition of the "Internet Official Protocol Standards" (STD 1) for the standardization state and status of this protocol. Distribution of this memo is unlimited.

Copyright Notice

Copyright (C) The IETF Trust (2007).

Abstract

This document defines a RADIUS (Remote Authentication Dial In User Service) attribute that carries an IPv6 prefix that is to be delegated to the user. This attribute is usable within either RADIUS or Diameter. 
1. Introduction

This document defines the Delegated-IPv6-Prefix attribute as a RADIUS [1] attribute that carries an IPv6 prefix to be delegated to the user, for use in the user's network. For example, the prefix in a Delegated-IPv6-Prefix attribute can be delegated to another node through DHCP Prefix Delegation [2].

The Delegated-IPv6-Prefix attribute can be used in DHCP Prefix Delegation between the delegating router and a RADIUS server, as illustrated in the following message sequence.

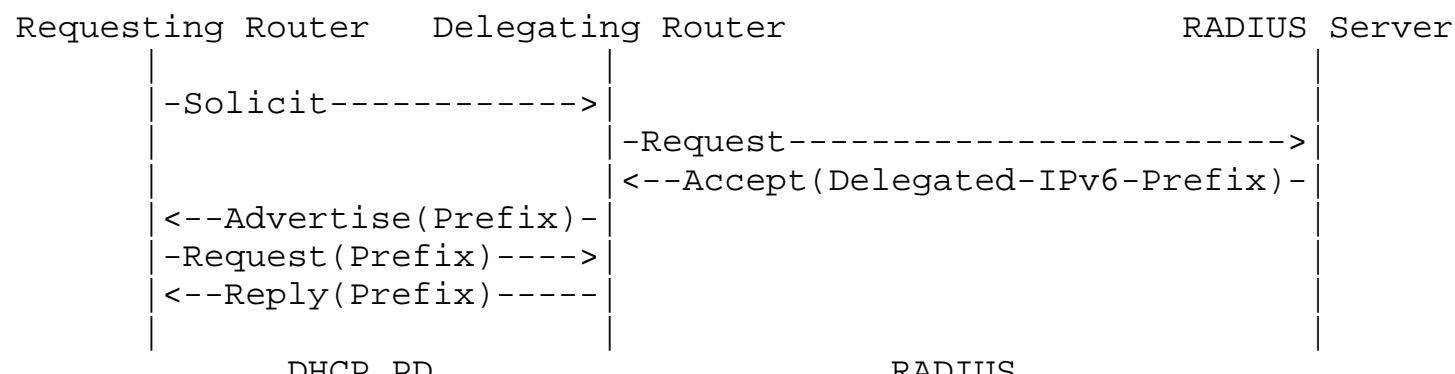

The Framed-IPv6-Prefix attribute [4] is not designed to support delegation of IPv6 prefixes to be used in the user's network, and therefore Framed-IPv6-Prefix and Delegated-IPv6-Prefix attributes may be included in the same RADIUS packet.

2. Terminology

The key words "MUST", "MUST NOT", "REQUIRED", "SHALL", "SHALL NOT", "SHOULD", "SHOULD NOT", "RECOMMENDED", "MAY", and "OPTIONAL" in this document are to be interpreted as described in RFC 2119 [3]. 


\section{Attribute Format}

The format of the Delegated-IPv6-Prefix is:

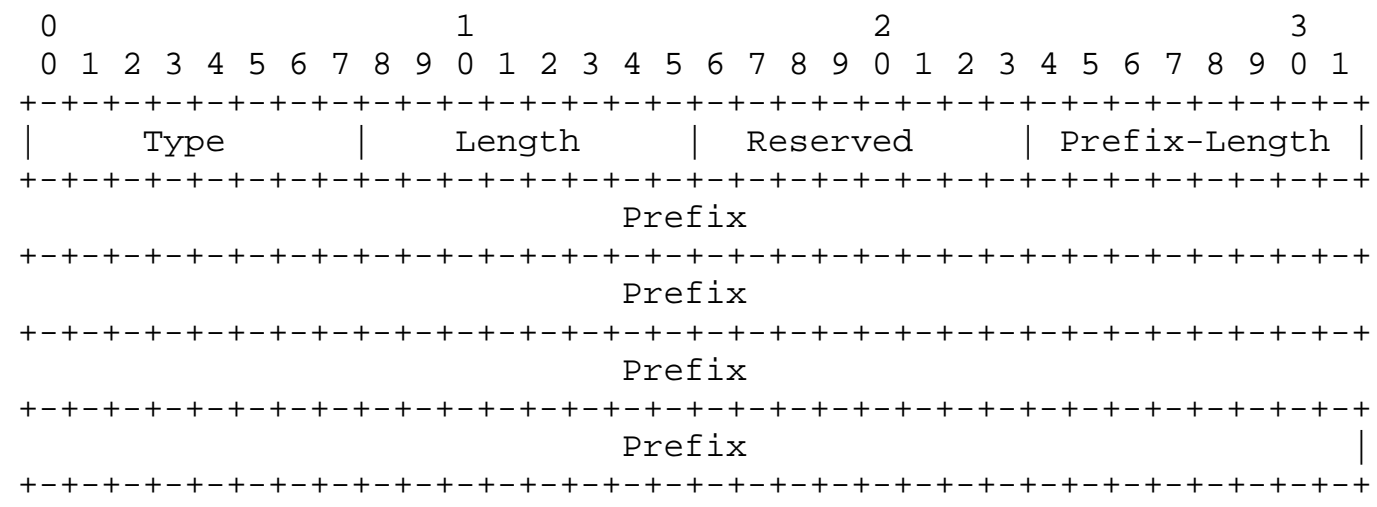

Type

123 for Delegated-IPv6-Prefix

Length

The length of the entire attribute, in bytes. At least 4 (to hold Type/Length/Reserved/Prefix-Length for a 0-bit prefix), and no larger than 20 (to hold Type/Length/ Reserved/PrefixLength for a 128-bit prefix)

Reserved

Always set to zero by sender; ignored by receiver

Prefix-Length

The length of the prefix being delegated, in bits. At least 0 and no larger than 128 bits (identifying a single IPv6 address)

Note that the prefix field is only required to be long enough to hold the prefix bits and can be shorter than 16 bytes. Any bits in the prefix field that are not part of the prefix MUST be zero.

The Delegated-IPv6-Prefix MAY appear in an Access-Accept packet, and can appear multiple times. It MAY appear in an Access-Request packet as a hint by the NAS to the server that it would prefer these prefix(es), but the server is not required to honor the hint. 
The Delegated-IPv6-Prefix attribute MAY appear in an AccountingRequest packet.

The Delegated-IPv6-Prefix MUST NOT appear in any other RADIUS packets.

4. Table of Attributes

The following table provides a guide to which attributes may be found in which kinds of packets, and in what quantity.

\begin{tabular}{|c|c|c|c|c|c|c|}
\hline Request & Accept & Reject & Challenge & $\begin{array}{l}\text { Accounting } \\
\text { Request }\end{array}$ & $\#$ & Attribute \\
\hline $0+$ & $0+$ & 0 & 0 & $0+$ & 123 & $\begin{array}{l}\text { Delegated-IPv6- } \\
\text { Prefix }\end{array}$ \\
\hline
\end{tabular}

The meaning of the above table entries is as follows:

0 This attribute MUST NOT be present.

$0+$ Zero or more instances of this attribute MAY be present.

0-1 Zero or one instance of this attribute MAY be present.

1 Exactly one instance of this attribute MUST be present.

1+ One or more of these attributes MUST be present.

5. Diameter Considerations

When used in Diameter, the attribute defined in this specification can be used as a Diameter AVP from the Code space 1-255, i.e., RADIUS attribute compatibility space. No additional Diameter Code values are therefore allocated. The data types of the attributes are as follows:

$$
\text { Delegated-IPv6-Prefix }
$$

Octetstring

The attribute in this specification has no special translation requirements for Diameter to RADIUS or RADIUS to Diameter gateways, i.e., the attribute is copied as is, except for changes relating to headers, alignment, and padding. See also RFC 3588 [5], Section 4.1, and RFC 4005 [6], Section 9.

The text in this specification describing the applicability of the Delegated-IPv6-Prefix attribute for RADIUS Access-Request applies in Diameter to AA-Request [6] or Diameter-EAP-Request [7].

The text in this specification describing the applicability of the Delegated-IPv6-Prefix attribute for RADIUS Access-Accept applies in Diameter to AA-Answer or Diameter-EAP-Answer that indicates success. 
The text in this specification describing the applicability of the Delegated-IPv6-Prefix attribute for RADIUS Accounting-Request applies to Diameter Accounting-Request [6] as well.

The AVP flag rules [5] for the Delegated-IPv6-Prefix attribute are:

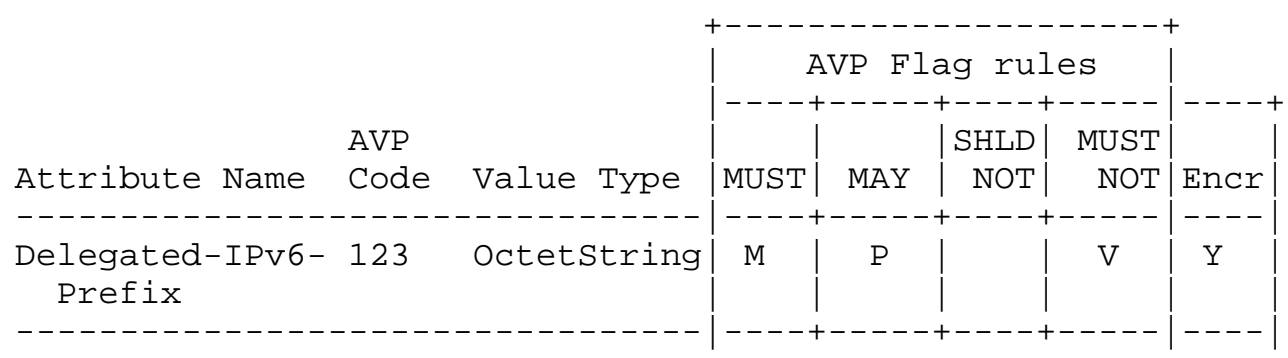

6. IANA Considerations

IANA assigned a Type value, 123, for this attribute from the RADIUS Attribute Types registry.

7. Security Considerations

Known security vulnerabilities of the RADIUS protocol are discussed in RFC 2607 [8], RFC 2865 [1], and RFC 2869 [9]. Use of IPsec [10] for providing security when RADIUS is carried in IPv6 is discussed in RFC 3162 .

Security considerations for the Diameter protocol are discussed in RFC 3588 [5].

8. References

8.1. Normative References

[1] Rigney, C., Willens, S., Rubens, A., and W. Simpson, "Remote Authentication Dial In User Service (RADIUS)", RFC 2865, June 2000 .

[2] Troan, O. and R. Droms, "IPv6 Prefix Options for Dynamic Host Configuration Protocol (DHCP) version 6", RFC 3633, December 2003 .

[3] Bradner, S., "Key words for use in RFCs to Indicate Requirement Levels", BCP 14, RFC 2119, March 1997. 


\subsection{Informative References}

[4] Aboba, B., Zorn, G., and D. Mitton, "RADIUS and IPv6", RFC 3162, August 2001 .

[5] Calhoun, P., Loughney, J., Guttman, E., Zorn, G., and J. Arkko, "Diameter Base Protocol", RFC 3588, September 2003.

[6] Calhoun, P., Zorn, G., Spence, D., and D. Mitton, "Diameter Network Access Server Application", RFC 4005, August 2005.

[7] Eronen, P., Hiller, T., and G. Zorn, "Diameter Extensible Authentication Protocol (EAP) Application", RFC 4072, August 2005 .

[8] Aboba, B. and J. Vollbrecht, "Proxy Chaining and Policy Implementation in Roaming", RFC 2607, June 1999.

[9] Rigney, C., Willats, W., and P. Calhoun, "RADIUS Extensions", RFC 2869, June 2000 .

[10] Kent, S. and K. Seo, "Security Architecture for the Internet Protocol", RFC 4301, December 2005 .

Authors' Addresses

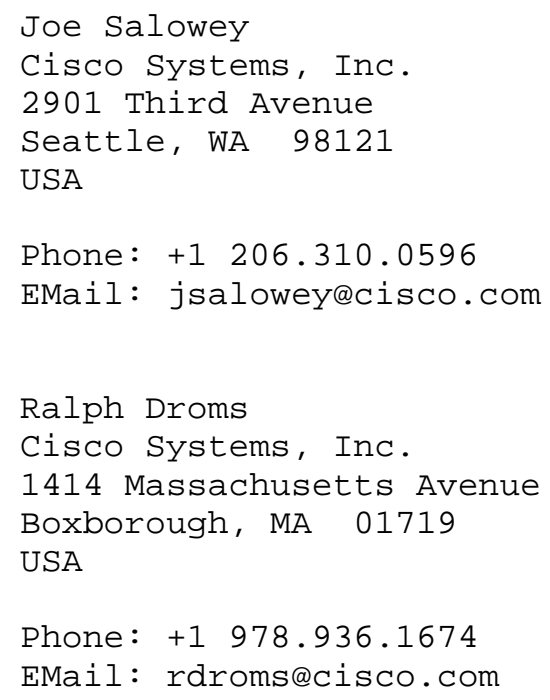


Full Copyright statement

Copyright (C) The IETF Trust (2007).

This document is subject to the rights, licenses and restrictions contained in BCP 78, and except as set forth therein, the authors retain all their rights.

This document and the information contained herein are provided on an "AS IS" basis and THE CONTRIBUTOR, THE ORGANIZATION HE/SHE REPRESENTS OR IS SPONSORED BY (IF ANY), THE INTERNET SOCIETY, THE IETF TRUST AND THE INTERNET ENGINEERING TASK FORCE DISCLAIM ALL WARRANTIES, EXPRESS OR IMPLIED, INCLUDING BUT NOT LIMITED TO ANY WARRANTY THAT THE USE OF THE INFORMATION HEREIN WILL NOT INFRINGE ANY RIGHTS OR ANY IMPLIED WARRANTIES OF MERCHANTABILITY OR FITNESS FOR A PARTICULAR PURPOSE.

Intellectual Property

The IETF takes no position regarding the validity or scope of any Intellectual Property Rights or other rights that might be claimed to pertain to the implementation or use of the technology described in this document or the extent to which any license under such rights might or might not be available; nor does it represent that it has made any independent effort to identify any such rights. Information on the procedures with respect to rights in RFC documents can be found in BCP 78 and BCP 79 .

Copies of IPR disclosures made to the IETF Secretariat and any assurances of licenses to be made available, or the result of an attempt made to obtain a general license or permission for the use of such proprietary rights by implementers or users of this specification can be obtained from the IETF on-line IPR repository at http://www.ietf.org/ipr.

The IETF invites any interested party to bring to its attention any copyrights, patents or patent applications, or other proprietary rights that may cover technology that may be required to implement this standard. Please address the information to the IETF at ietf-ipreietf.org.

Acknowledgement

Funding for the RFC Editor function is currently provided by the Internet Society. 